\title{
The effect of blindness on DRL-20 performances exhibited by animals with hippocampal destruction'
}

\author{
LEONARD W. SCHMALTZ AND ROBERT L. ISAACSON, \\ PSYCHOLOGICAL LABORATORIES, THE UNIVERSITY OF \\ MICHIGAN, Ann Arbor, Mich. 48104
}

Unoperated rats and $S s$ with bilateral hippocampal destruction were peripherally blinded and tested for acquisition of a DRL-20 (differential reinforcement for low rates of responding) schedule of reinforcement. The performance of the blinded $S s$ was compared with that of nonblinded unoperated $S s$ and $S s$ with hippocampal or neocortical damage. Peripherally blinded hippocampal Ss received significantly more reinforcements and bar pressed significantly less than nonblind $S$ s with similar lesions. Blindness in unoperated rats was found to have no effect on response rates but did diminish the number of reinforcements received on the DRL schedule.

In three previous studies (Clark \& Isaacson, 1965; Schmaltz \& Isaacson, 1966a; Haddad \& Rabe, 1968) it was reported that rats with bilateral hippocampal destruction were impaired in the acquisition of a DRL-20 (differential reinforcement for low rates of responding) schedule of reinforcement.

On several occasions in our laboratory we observed that accidental bilateral damage to the optic tract, lateral geniculate nucleus, or visual radiations seemed to mitigate, to some extent, the debilitating effects of hippocampal destruction in both rats and cats. In the present study, we attempted to investigate systematically the relationship between peripheral blindness, hippocampal lesions, and DRL-20 performances.

\section{METHOD}

Subjects and Apparatus. The Ss were 16 naive male Long-Evans rats approximately 120 days old at the start of the experiment. A Grason-Stadler (Model E3125A-300) operant chamber, which discharged a 45-mg food pellet as reinforcement, was used.

Surgery. The surgical procedures used in performing the bilateral hippocampal lesions (eight Ss) have been described in detail previously (Isaacson Douglas, \& Moore, 1961).

All $\mathrm{Ss}$ in the present study (eight hippocampal Ss and eight normal Ss) were peripherally blinded. This was accomplished by sectioning the optic nerves just behind the globus. The occulomotor muscles and eye were left in place following the sectioning of the nerve. This procedure was carried out in the brain-lesioned animals at the time of hippocampal destruction.

Following surgery, the Ss were given a one-month recovery period. During the first two weeks, they were given ad lib diets and weighed daily. They were then placed on a $23 \mathrm{~h}$ deprivation schedule. At the end of the two week period, all Ss' weights had stabilized at approximately $90 \%$ of their ad lib weights.

Postoperative Testing. All Ss were shaped to the bar press response. They were given 20 daily CRF (continuous reinforcement) sessions of $30 \mathrm{~min}$ duration. They were then given 16 daily DRL-20 sessions of $30 \mathrm{~min}$ duration.

Histological Evaluation. Following the experiment, all Ss were sacrificed, intracardially perfused with $10 \%$ formalin, and the brains removed.

The brains of those Ss which sustained hippocampal lesions were cut at $20 \mu$ in paraffin. Appropriate sections were mounted and stained with thionin.

The brains of the unoperated, but peripherally blinded, Ss were cut at $25 \mu$. All sections were prepared with a Marchi stain for degenerating myelin (Davenport, 1960). Alternate sections were counter-stained with thionin.

The purpose of the present study was to evaluate the effect of peripheral blindness upon the impairments in performance found by hippocampal destruction. Accordingly, the performances of the two groups of peripherally blinded Ss of the present study were compared with the performances of three groups of Ss of identical strain, sex, age, weight, and past histories, as well as surgical and training procedures (Schmaltz \& Isaacson, 1966a). In this earlier study Ss in the cortical group had the dorsolateral neocortex overlying the hippocampus removed. The hippocampus itself was left in tact.

Histological reconstructions of the hippocampal lesions in the present study were prepared in our usual manner (Schmaltz \& Isaacson, 1966a, $1966 \mathrm{~b}, 1967)$. There were no significant differences between the hippo- campal lesions of our earlier study (Schmaltz \& Isaacson, 1966a) and those in the present work. The mean percentages of hippocampal destruction, for example, were $59 \%$ and $63 \%$, respectively.

All of the brains (stained according to the Marchi technique) of the unoperated Ss in the present study showed bilateral degeneration of the optic tract. We were unable to detect significant degeneration in any other brain areas.

\section{RESULTS AND DISCUSSION}

CRF Performance. During the $20 \mathrm{CRF}$ sessions, no statistical difference in the number of bar presses produced by the five groups of animals was found. A two-way analysis of variance showed no group, sessions, or interaction effects.

DRL-20 Performance. Figure 1 presents the median number of responses emitted (top) and reinforcements received (middle) during the DRL-20 sessions. A two-way analysis of variance indicated significant group effects and individual comparisons between groups were made as outlined by Winer (1962). A summary of the $F$ values is given in Table 1 . At the bottom of Fig. 1, the median ratios of rewarded responses to total responses made in each session are shown.

Peripheral blindness markedly reduced the number of bar presses made by Ss with hippocampal damage and increased the
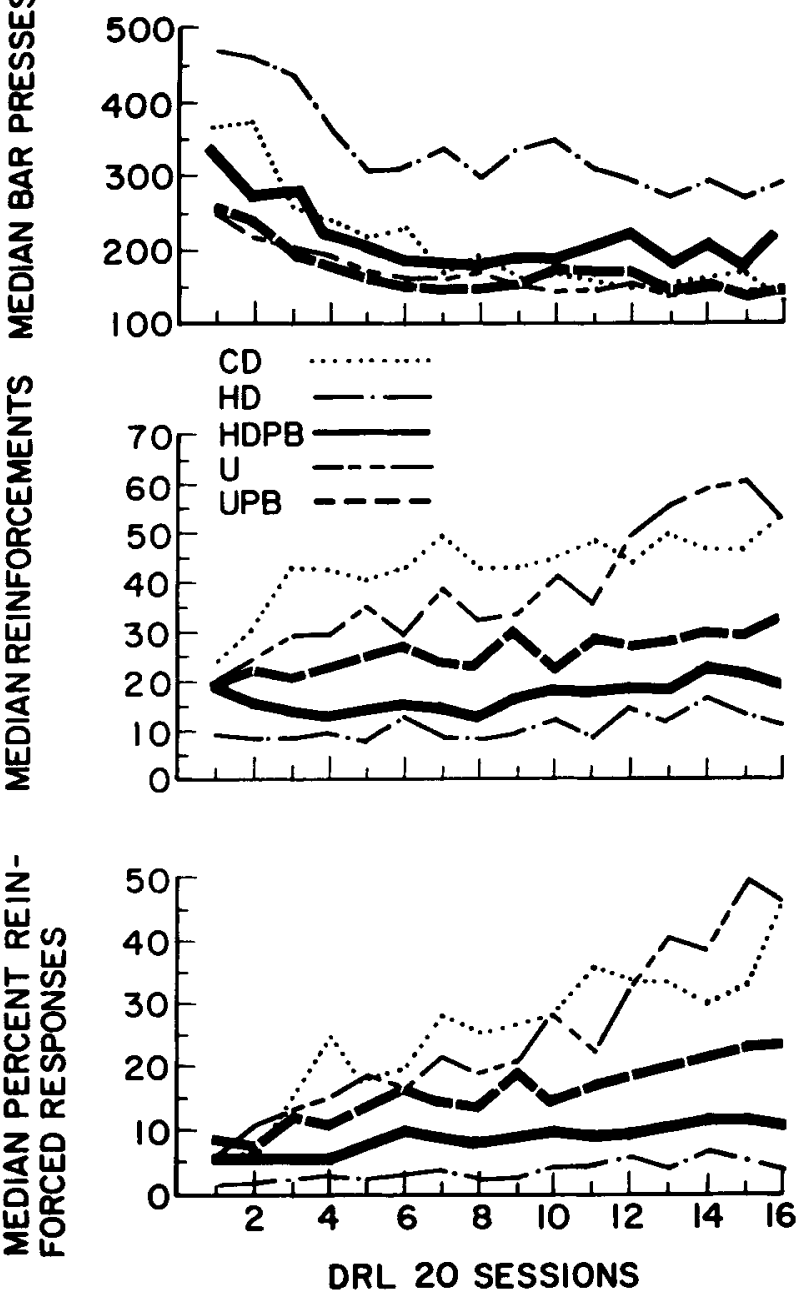

Fig. 1. Performance records of the Ss while on the DRL schedule. See Table 1 for explanation of legend. 
Table 1*

Summary of the Statistical Evaluation of the Data

$\begin{array}{llll}\text { Reinforcements: } & \text { (Main Effects) } & \\ \text { Group Effect: } & F=34.20 ; \quad \text { Sessions Effect: } & F=13.58 ; \\ & \text { Interaction Effect: } \quad F=283.57 & \\ \text { Responses: } & \text { (Main Effects) } & \\ \text { Group Effect: } & F=11.67 ; \quad \text { Sessions Effect: } & F=32.08 ; \\ & \text { Interaction Effect: } \quad F=2.39 & \end{array}$

Individual Comparisons**

\begin{tabular}{|c|c|c|c|c|c|c|}
\hline & & $\mathrm{U}$ & $\mathrm{CD}$ & UPB & $\mathrm{HD}$ & HDPB \\
\hline Unoperates & (U) & $\mathbf{X}$ & - & 9.23 & 21.95 & 23.35 \\
\hline $\begin{array}{l}\text { Cortical } \\
\text { Destruction }\end{array}$ & (CD) & - & $X$ & 16.28 & 46.03 & 35.07 \\
\hline $\begin{array}{l}\text { Jnoperates } \\
\text { Peripherally } \\
\text { Blinded }\end{array}$ & (UPB) & - & - & $X$ & 8.83 & 17.07 \\
\hline $\begin{array}{l}\text { Hippocampal } \\
\text { Destruction }\end{array}$ & (HD) & 22.08 & 22.04 & 33.21 & $\mathrm{X}$ & 2.75 \\
\hline Hippocampal & (HDPB) & - & - & - & 20.19 & $X$ \\
\hline
\end{tabular}

${ }^{*}$ All $F$ values listed are significant at the .01 level, except for the value 2.75 which is significant at the .05 level. A dash indicates that the difference is not statistically significant.

**The numbers to the right of the diagonal of " $X$ " are $F$ values for the individual comparisons between groups for the number of reinforcements received. Those to the left are for the number of responses made.

number of reinforcements received by these animals. Blindness failed to affect the number of bar presses emitted by the unoperated animals, but it did adversely affect the number of reinforcements they received. The favorable effects on the DRL-20 performance of blind hippocampally lesioned Ss leads to certain difficulties in the interpretation of the effects of hippocampal destruction.

Our most recent view of the hippocampal deficit was that the lesion led to an exaggerated responsiveness to changes in reinforcement contingencies as well as to other significant alterations in the environment (Schmaltz \& Isaacson, 1967). This view is similar to the suggestion of Kluver (1965), Haddad \& Rabe (1968), and Swanson \& Isaacson (1967). The fact that blindness in hippocampally damaged Ss reduces the number of bar presses to that of Ss with only neocortical damage, and nearly. to that of animals with intact brains, suggests that this overresponsiveness is dependent, in part, upon usual levels of sensory stimulation. It is possible that reductions of afferent information in other modalities could produce an equally dramatic change in response rate on the part of Ss with hippocampal damage.

If the reduction of sensory input over several modalities were to improve the performance of animals with hippocampal damage, one might wish to speculate that the exaggerated responsiveness of hippocampally damaged Ss was due to a reduction of inhibition on systems of the diencephalon and midbrain associated with energizing behavior, and that the reduction of sensory input tends to reduce activity in these energizing circuits.

An interesting and unexpected result of this experiment was that the number of reinforcements received by blinded hippocampal Ss was not nearly as large as might be anticipated from the sizeable reduction in response rate. While there was a significant improvement over the performance on nonblind Ss with hippocampal damage, the blinded operates performed rather poorly and significantly worse than blinded animals without brain damage. This suggests that the impairment on the DRL-20 schedule produced by hippocampal damage is due to several factors, one related to the exaggerated rate of responding, which is at least partially reduced by blindness, and a second, as yet unknown, factor, independent of response rate.

\section{REFERENCES}

CLARK, C. V., \& ISAACSON, R. L. Effect of bilateral hippocampal ablation on DRL performance. J. comp. physiol. Psychol., 1965, 59, 137-140.

DAVENPORT, H. A. Histological and histochemical techniques. Philadelphia: W. B. Saunders, 1960.

HADDAD, R. K., \& RABE, A. Effect of selective hippocampal lesions in the rat on DRL-20 acquisition. J. psychol. Studies, in press.

ISAACSON, R. L., DOUGLAS, R. J., \& MOORE, R. Y. The effect of radical hippocampal ablation on acquisition of avoidance responses. J. comp. physiol. Psychol., 1961, 54, 625-628.

KLUVER, H. Neurobiology of normal and abnormal perception. In P. H. Hoch \& J. Zubin (Eds.), Psychopathology of perception, New York: Grune \& Stratton, 1965.

SCHMALTZ, L. W., \& ISAACSON, R. L. The effects of preliminary training conditions upon DRL-20 performance in the hippocampectomized rat. Physiol. Behav., 1966a, 175-182.

SCHMALTZ, L. W., \& ISAACSON, R. L. Postoperative retention of a DRL-20 schedule by hippocampectomized and partially neodecorticate rats. J. comp. physiol. Psychol, 1966b, 128-132.

SCHMALTZ, L. W., \& ISAACSON, R. L. Effect of bilateral hippocampal destruction on the acquisition and extinction of an operant response. Physiol. Behav., 1967, 291-298.

SWANSON, A. M., \& ISAACSON, R. L. Hippocampal ablation and performance during withdrawal of reinforcement. $J$. comp. physiol. Psychol, 1967, 64, 30-35.

WINER, B. J. Statistical principles in experimental design. New York: McGraw-Hill, 1962.

\section{NOTE}

1. This study was supported in part by a grant from the National Institute of Health to Robert L. Isaacson (NIH MH-11285-02). 